\title{
N-doped Cycloparaphenylenes: Tuning Electronic Properties for Applications in Thermally Activated Delayed Fluorescence
}

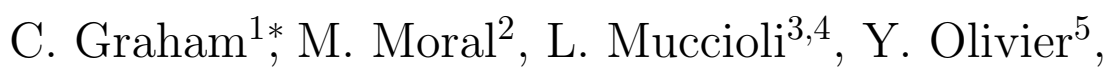
A. J. Pérez-Jiménez ${ }^{1}$, and J. C. Sancho-García ${ }^{1 \dagger}$

${ }^{1}$ Department of Physical Chemistry, University of Alicante, E-03080 Alicante, Spain

2 Renewable Energy Research Institute, University of Castilla-La Mancha, E-02071 Albacete, Spain

${ }^{3}$ Department of Industrial Chemistry "Toso Montanari", University of Bologna, IT-40136, Italy

${ }^{4}$ Institut des Sciences Moléculaires, UMR 5255, University of Bordeaux, F-33400 Talence, France

${ }^{5}$ Laboratory for Chemistry of Novel Materials, University of Mons, B-7000 Mons, Belgium

November 20, 2017

* On leave from the School of Chemistry, University of Edinburgh, Edinburgh, U.K.

†E-mail: jc.sancho@ua.es 


\begin{abstract}
We theoretically characterise a series of substituted cycloparaphenylene nanohoops to study the effect of incorporating an electronwithdrawing group into their cyclic structure. We systematically vary the nature, position, and number of nitrogen-containing acceptor groups in both neutral (pyridine) and charged forms (pyridinium and methylpyridinium) to provide insights into how this functionalization affects the structural, electronic, and optical properties of these systems. We focus also on the singlet-triplet energy difference, with low values found, which might pave the way to further applications in the field of devices for light-emitting applications providing a potential class of TADFbased emitters.
\end{abstract}

Key words: Donor-acceptor CycloParaPhenylenes, optoelectronic properties, Thermally Activated Delayed Fluorescence, DFT and TD-DFT. 


\section{Introduction}

Organic Electronics is a field of rapid growth attracting interest from interdisciplinary domains including Electronics \& Engineering, Chemistry, Physics, and Materials Science, not to mention the great commercial interest towards its applications $[1,2]$. The facile tunability of organic molecules has paved the way for a wealth of organic-based materials to be synthesised with novel key properties. Already, small organic molecules find their place in photovoltaic cells $[3,4]$, light-emitting transistors [5-9] and diodes [10-12], to name just a few of these applications. The flexibility of organic materials over inorganic has enabled the birth of the "soft electronics" market, including devices that can twist, bend or mould to any surface or textile. With so many possibilities arising from unique properties of organic molecules, the future of organics appears bright: not only do organic materials promise more innovative technologies but also are far more sustainable (organic molecules are abundant, easily synthesised and potentially recyclable). Therefore, the motivations for development of organic-based devices are still valid: (i) to surpass the applicability of silicon-based devices by expanding the functionality of electronics; and (ii) to be more sustainable in terms of energy efficiency while manufactured using low resource usage methods.

In particular this study consists in the deployment of a family of not yet fully explored organic nanorings, an emerging subclass of macrocycles [13-18] with great possibilities for their application as active materials in optoelectronic devices. The cyclic topology of these systems already presents intriguing properties due to its unique combination of strain and bending, and the radial arrangement of $\pi$-orbitals; contrarily to the case of linear oligomers most studied up to date. Actually, some nanorings have recently been stud- 
ied, displaying competitive charge-transport and photophysical properties, at least from the theoretical point of view [19]. Complementarily, there have been recent advances towards other related $\pi$-extended systems [20-22] which may afford a fine-tuned and fully controlled synthesis of short carbon nanotubes from these molecular building blocks, to possibly contribute to a new realm of technology with light weight, low cost, flexible electronics. To realise this potential, the often conflicting design protocols for efficient charge injection and transport, light emission upon charge recombination, and exciton diffusion, need to be overcome. Significant efforts in this direction have been reported in last years, but to our knowledge these processes has not been systematically explored in the context of small nanorings. In this regard, we theoretically investigate here Donor-Acceptor (D-A) CycloParaPhenylene (CPP) derivatives [23-30], whose molecular design strategy is presented in Figure 1, and specifically a large set of Nitrogen-doped (N-doped) CPPs. Note that the choice of the systems is motivated by recent experimental achievements [31] but extended to other substitution patterns for which the D-A moieties might be of key importance in steering new mechanisms for light emission (vide infra). Figure 2 shows the chemical structure of all the compounds studied.

The manuscript is organised into the following sections. We will outline first (Section 2) the set of theoretical models selected for studying accurately these systems, in both their ground- and excited-states, and in gasor solution-phase. Next we address in detail (Subsections 3.1 and 3.2) the structural and optoelectronic properties of a set of N-doped CPPs, derived from the pristine [8] CPP (where square brackets indicate the number of benzene rings of the CPP system) by varying the position, type and number of 
the acceptor groups. In doing so, we hope to shed light on the structureproperty relationships that govern these challenging systems once functionalized. Then we also conduct (Subsection 3.3) a size-dependent study of $\mathrm{N}$-doped CPPs, including other members such as [6]CPP and [10]CPP, analyzing if the structural and optoelectronic effects of N-doping change with the diameter of the nanorings. Next we study their optical properties (Subsection 3.4), and then consider (Subsection 3.5) the suitability of these nanorings to exhibit light emission via a novel Thermally Activated Delayed Fluorescence (TADF) mechanism. Note that the design of organic semiconductors with TADF properties, which are primarily based on D-A moieties [32,33], has seen enormous progress in recent years [34-37]. In this regard, we evaluate the impact of the acceptor groups on reducing the singlet-triplet energy gap, and we concomitantly employ some metrics for rationalizing the nature of these excited-states, aiming at evaluating their potential as emitters in OLED applications.

\section{Theoretical framework}

\subsection{General issues}

All the calculations reported here were done with the G09 package [38], using Gabedit [39], Multiwfn [40], and the NANCY_EX package [41,42] for postprocessing of the results. To model the electrostatic effects arising in solution, and thus to truly compare whenever possible with the set of experimental results mostly performed in acetonitrile or in DiChloroMethane (DCM), for charged nanohoops we employ the Conductor-like Polarisation Continuum Model (CPCM) [43,44] according to recent recommendations [45], while the neutral compounds are treated by the standard PCM model [46]. The cost- 
effective basis set 6-31G* was fixed for most of the calculations reported here. The larger def2-TZVP basis set $[47,48]$ was also used, as a sanity check in some cases, to verify the completeness of the $6-31 \mathrm{G}^{*}$ basis set, for instance, for the TD-DFT calculations.

\subsection{Ground-state calculations}

Due to the expected interplay between large size and concurring stereoelectronic effects arising from CPP strained cyclic topology, Density Functional Theory (DFT) is the most appropriate theoretical method (vide infra) for studying accurately their properties at a reasonable computational cost. We preliminarily selected some hybrid exchange-correlation flavours, ordered for their increasing weight (in \%) of Hartree-Fock (HF) like exchange, to address the possible impact of this technicality on the ground-state properties: M06-L (0 \%) [49], B3LYP (20 \%) [50,51], M06-2X (54 \%) [49], and M06-HF $(100 \%)$ [49], as well as the range-separated $\omega$ B97XD [52]. After the initial benchmarking done, we also applied the -D3(BJ) dispersion correction $[53,54]$ to take into account possible missing intra-molecular (long-range) interactions. We have always verified that all real frequencies are obtained in all cases for the optimized ground-state geometries.

\subsection{Excited-state calculations}

Figure 3 sketches all the processes involved, using a simplified representation of the relevant potential energy surfaces as a function of a generalized coordinate $Q$. Note that we are basically interested in disclosing the underlying structure-property guidelines for these materials, and not in pursuing 
the benchmark of theoretical methods against large datasets; nonetheless, based on recent key studies of excited-state properties $[55,56]$, we have also selected the PBE0 functional [57] for the TDA-DFT part in addition to the functionals employed for the ground-state part. The main difference between this and a previous study, which also included some calculations for a subset of the N-doped CPPs [31], relies not only on benchmarking of theoretical methods but also on unexplored excited-state light-emitting properties (vide infra).

Vertical excitation energies, from the ground-state optimized geometries to the first lowest singlet $E_{v}\left(S_{1}\right)$ or triplet $E_{v}\left(T_{1}\right)$ excited-states, were calculated within the Time-Dependent (TD-)DFT approximation using the Tamm-Dancoff (TDA-)DFT linear-response approach [58,59], which provides improved accuracy specially for triplet state energies [60-62] and keeps also other advantages (e.g. lower cost) for further applications to large systems [63]. The difference between the vertical singlet and triplet absorption energies gives the corresponding (vertical) singlet-triplet splitting, $\Delta E_{v}^{S T}$. We also compute in some cases the adiabatic excitation energies, $E_{a}\left(S_{1}\right)$ and $E_{a}\left(T_{1}\right)$, which accounts for the reorganization energy $(\lambda)$ upon relaxation of the excited-state geometries, through the corresponding TDA-DFT gradients. The energy difference between these two states is denoted as $\Delta E_{a}^{S T}$. The optimized lowest triplet excited-state was also accessed (in some cases) by spin-unrestricted calculations and yielded the same result than TDA-DFT (within $\pm 0.05 \mathrm{eV}$ ) for the $E_{a}\left(T_{1}\right)$ values. 


\section{Results and discussion}

\subsection{Study of the structural effects of acceptor sub- stituents into [8] CPP}

We evaluate first the structural effects of substituting a $\mathrm{CH}$ by a $\mathrm{N}$ atom or a $\mathrm{N}$-containing $\left(\mathrm{NH}^{+}\right.$or $\left.\mathrm{NCH}_{3}^{+}\right)$group by calculating: (i) the dihedral angle between adjacent phenyl units; and (ii) the Bond Length Alternation (BLA) for all phenyl units, measuring the degree of $\pi$-conjugation along the rings of the backbone. Note that we refer in the following to the doping of the molecule when this substitution is done, even when replacing a $\mathrm{CH}$ by a $\mathrm{N}$ atom creates an isoelectronic (and closed-shell) compound. We discuss as a matter of illustration only a subset (see Figure 2) of the systems tackled: 1,15-diaza-[8]CPP 2, H,H-1,15-diaza-[8] $\mathrm{CPP}^{2+} \mathbf{6}$, and N,N-dimethyl1,15-diaza-[8] $\mathrm{CPP}^{2+} \mathbf{8}$, with the corresponding results presented in Table 1 and compared with the [8]CPP molecule.

The architecture of CPPs confers to the systems a unique cyclic structure of strained, $\mathrm{sp}^{2}$ hybridized, and para-linked phenyl rings. Generally speaking, the most energetically favoured conformation found in real samples [64] is that which reduces ortho, ortho' steric interactions between adjacent phenyl rings while maximising $\pi$-conjugation. Regarding the dihedral angles, and independently of the exchange-correlation functional applied, the unsubstituted [8]CPP assumes a staggered orientation with adjacent phenyl rings cantering alternately by about $\pm 30^{\circ}$, with a dihedral angle slightly reduced (i.e. by up to $5^{\circ}$ ) with respect to that of the corresponding linear

oligomer $[65,66]$. The same configuration is also retained by N-doped CPPs, although more planar angles are predicted due to a reduced steric hindrance between the nitrogen lone pair and the adjacent aryl $\mathrm{C}-\mathrm{H}$ group (Table 1). 
The evolution of the BLA calculated by all the assessed DFT methods is shown in Figure 4. All functionals give qualitatively consistent results and, as expected from the absence (full presence) of HF-like exchange, the M06-L (M06-HF) predicts the lowest (highest) values according to the overdelocalization error (full localization) of this functional. Since the BLA and optical excitations are properties intimately connected $[67,68]$, these methods are expected to underestimate (overestimate) exciton energies [69]. The M06-2X and $\omega$ B97XD methods behave similarly, specially for the 1,15-diaza-[8]CPP 2 and N,N-dimethyl-1,15-diaza-[8]CPP ${ }^{2+} \mathbf{8}$ cases, while B3LYP-D3(BJ) lies between M06-L and M06-2X, following the increasing weight of the HF-like exchange introduced into the method [70]. We also note that the $\omega \mathrm{B} 97 \mathrm{XD}$ method had been shown previously to overestimate systematically the experimental absorption maxima for the set of increasingly longer [6-12] CPPs [71], and we will thus rely in the following in the B3LYP-D3(BJ) optimized geometries as a compromise. We calculate with this method a diameter of $11.0 \pm 0.5 \AA$ for molecule $\mathbf{1}$, to be compared with an experimental (X-ray) value of $10.9 \pm 0.2 \AA$ [31], and that the precise location of the $\mathrm{N}$ atom could not be experimentally resolved, which precludes a more detailed comparison between experimental and theoretical results for individual bond lengths.

\subsection{Electronic properties of $\mathrm{N}$-doped [8]CPPs}

We inspect in detail the energy and shape of the frontier molecular orbitals of cyclic compounds, at the B3LYP/6-31G* level, and compare with those of the pristine [8]CPP molecule and of their corresponding N-doped linear oligomers. Figure 5 shows the trend in the energy difference between the 
Highest Occupied (HO) and the Lowest Unoccupied (LU) Molecular Orbital (MO), and how it is reduced in all cases with respect to that of the undoped [8]CPP, with a more pronounced impact for the protonated or alkylated nanohoops. Actually, we can see that further increasing the content of $\mathrm{N}$ in molecules 1-3 has small effects on the HOMO (up to $0.1 \mathrm{eV}$ ) or LUMO (up to $0.3 \mathrm{eV}$ ) energies, which are found delocalized over the whole backbone, and thus on the corresponding gap. Interestingly, the energy of the HOMO is only weakly altered for the whole set of molecules studied, ranging between 5.1 and $5.6 \mathrm{eV}$ for most of the cases unless for the multiply charged compounds $\mathbf{6}$ and $\mathbf{9}$, but the LUMO energy is dramatically stabilized by up to 1 $\mathrm{eV}$ in some cases when going from the neutral to any of the charged $\mathrm{N}$-doped CPPs. This trend fully agrees with the experimental decrease in the cathodic peak potential for the reduction of compounds $\mathbf{1 - 3}$ and $\mathbf{7 - 8}$, according to a recent study [31].

We now compare cyclic vs. linear N-doped paraphenylenes, CPPs and LPPs respectively, using the same computational protocol for both. The increased conjugation of the former (evidenced for instance by lower BLA values in a fewer rings) is consistent with a lower HOMO-LUMO gap (e.g. $3.33 \mathrm{eV}$ and $3.76 \mathrm{eV}$ calculated here, respectively, for aza-[8]CPP $\mathbf{1}$ and aza-[8]LPP). The narrowing of the HOMO-LUMO gap $(2.5-2.7 \mathrm{eV})$ for the charged cyclic compounds was also observed for a subset of the parent Ndoped LPPs, being for instance now of 2.55 and $2.80 \mathrm{eV}$ for aza-[8]CPP ${ }^{+} 4$ and aza- $[8] \mathrm{LPP}^{+}$, respectively.

Actually, for molecules 4-9, the HOMO is evenly distributed over the electron-rich phenylene backbone, the moiety acting as a donor, whereas 
the LUMO is located mainly on the N-(methyl)pyridinium core, acting thus as the acceptor, resulting in a pronounced intramolecular donor-acceptor orbital separation. Only in the triply-alkylated nanohoop 9, where the acceptor groups are evenly positioned around the nanohoop, they result in a more extended distribution of the LUMO orbital compared to other cases. Summarizing, the incorporation of a neutral pyridine moiety has a negligible effect compared to insertion of pyridinium and methylpyridinium units, both on the LUMO energies and on the intramolecular HOMO-LUMO separation, a fact that could be further explored (vide infra) for promoting new light emission mechanisms.

\subsection{Increasingly longer $\mathrm{N}$-doped $[n] \mathrm{CPPs}$}

We subsequently look at the effect of decreasing/increasing the number $(n)$ of aromatic rings of the N-doped nanohoop on the HOMO-LUMO orbitals for a subset of representative geometries, that is, aza- $[n] \mathrm{CPP}, \mathrm{H}$-aza$[n] \mathrm{CPP}^{+}$, and N-methylaza- $[n] \mathrm{CPP}^{+}$compounds, with $n=6,8$, and 10, and their corresponding linear oligomers (see Figure 6). Note that if $n=8$, these compounds correspond to numbers 1, 4, and $\mathbf{7}$ in Figure 2 before, and that again the B3LYP/6-31G* level of theory is used. As is known from literature, the CPPs have decreasing HOMO energies and increasing LUMO energies (increasing band gap) with increasing molecular size, opposite to linear oligomers [72]. For example, the N-methylaza- $[n] \mathrm{CPP}^{+}$series, with increasing molecular size, exhibit an increase in the HOMO-LUMO gap from 2.57 to $2.74 \mathrm{eV}$ for the N-methylaza- $[6] \mathrm{CPP}^{+}$to N-methylaza- $[10] \mathrm{CPP}^{+}$cases respectively, reaching rapidly the saturation values. However, the opposite trend is observed in the linear oligomers, with a decreasing HOMO-LUMO 
gap from 3.18 to $2.92 \mathrm{eV}$, respectively.

Furthermore, it seems that the aforementioned size-scaling electronic properties for CPPs are less prominent with increasing acceptor strength (pyridine $<$ methylpyridinium $<$ pyridinium). Whereas for the aza- $[n] \mathrm{CPP}$ series of compounds, the HOMO-LUMO gap increases monotonically from 2.87 to $3.37 \mathrm{eV}$ when going from the aza-[6]CPP to the aza-[10]CPP system, as it also happens for the unsubstituted $[n]$ CPPs, the gap does not significantly alters (up to 0.1-0.2 eV) in the other two cases, reflecting the strong localization of both frontier molecular orbitals in spatially separated fragments of the molecule. Thus, the conclusions drawn before about the impact of inserting pyridinium and methylpyridinium units are expected to also approximately hold, independently of the nanoring size, as it was also recently inferred experimentally after comparing aza-[6] CPP with aza-[8] CPP and N-methylaza- $[6] \mathrm{CPP}^{+}$with N-methylaza- $[8] \mathrm{CPP}^{+}[73]$. The same trend regarding the evolution of the HOMO-LUMO gap is also found for the N-doped linear paraphenylenes, although to a lesser extent due to edge effects: the gap decreases monotonically from 3.98 to $3.81 \mathrm{eV}$ for the aza-[6]LPP and aza-[10]LPP cases, and changes up to $0.1-0.3 \mathrm{eV}$ in the other cases, again reflecting some localization of both frontier molecular orbitals in spatially separated fragments of the charged molecules.

\subsection{Optical properties of N-doped [8]CPPs}

The TDA-DFT approach was used to provide results and insights into the photophysical properties of the set of N-doped [8]CPPs. We remind first that all unsubstituted $[n]$ CPPs experimentally share a common absorption 
maximum at $340 \mathrm{~nm}$ [74], independently of solvents, which is perfectly reproduced here with the TDA-B3LYP/6-31G*//B3LYP-D3(BJ)/6-31G* protocol for the $[8] \mathrm{CPP}$ case (see Figure 7) although care with this functional must be considered for much longer oligomer sizes [75]. Nonetheless, this level of theory has been also used before for pristine $[n]$ CPPs and can thus help to compare with previously published results $[76,77]$. Thus, we tackle next the simulated UV-vis absorption spectra of all the D-A nanohoops 1-9, presented in Figure 7.

It can be immediately seen how for the neutral N-doped nanohoops 13 a major absorbance peak is also observed around $340-350 \mathrm{~nm}$, slightly red-shifted upon increasing the content of $\mathrm{N}$ in perfect agreement with experimental results $[31,78]$. Note that contrarily to what it was found before for the unsubstituted [8] CPP [71,72] for these N-doped [8]CPPs, the HOMOLUMO transition is not forbidden due to partial symmetry breaking induced by the presence of nitrogen, although the oscillator strength values are very low. Actually, for both [8]CPP and neutral N-doped nanohoops 1-3, the transition $\left(\pi \rightarrow \pi^{\star}\right)$ giving rise to the absorption maximum is composed of approximately equal combinations of one-electron excitations from HOMO1 to LUMO and HOMO to LUMO+1, and from HOMO-2 to LUMO and $\mathrm{HOMO}$ to $\mathrm{LUMO}+2$.

The spectra drastically changes for the charged compounds 4-9, see also Figure 7, displaying a larger number of peaks and shoulders and thus reflecting a more complicated manifold of one-electron transitions giving rise to the corresponding excitations. Since experimental results are available for compounds N-methylaza-[8] $\mathrm{CPP}^{+} 7$ and N,N-dimethyl-1,15-diaza-[8] $\mathrm{CPP}^{2+}$ 
8 [31], we include in Figure 8 the energy and shape of a subset of the molecular orbitals involved into the lowest-energy excitations for these systems, as well as the corresponding (non-negligible) oscillator strengths. Actually, the introduction of the electroactive groups breaks the degeneracy of the HOMO1/HOMO-2 and LUMO+1/LUMO+2 orbitals found in undoped $[n] \mathrm{CPPs}$, which helps explaining the shape of the spectra involving multiple allowed absorption bands. Note that a broad absorption spectrum is advantageous for photovoltaic applications [79], to harvest the largest possible number of photons.

We also investigate if the choice of the functional would have any influence on the results obtained so far by comparing the HOMO-LUMO energy absorptions of $\mathbf{7}$ and $\mathbf{8}$, situated experimentally at 460 and $554 \mathrm{~nm}$, respectively, with the theoretical results (M06-L, B3LYP, PBE0, M06-HF, and $\omega \mathrm{B} 97 \mathrm{XD}$ functionals). We also investigate the effect (if any) of enlarging substantially the basis set going from the $6-31 G^{*}$ to the def2-TZVP one, choosing for that the PBE0 model as a test case. Table 2 shows how the values are largely underestimated (overestimated) by the M06-L (M06-HF and $\omega \mathrm{B} 97 \mathrm{XD})$ methods, following the conclusions about the performance of these methods also achieved in previous sections upon inspecting the BLA values. We also note that the same trends are also kept for the rest of the compounds, 1-6 and 9, analyzed here. Interestingly, the TDA-PBE0 results are in reasonable agreement (within $0.1-0.3 \mathrm{eV}$ ) with the experimental values, followed closely by the TDA-B3LYP ones; the use of the def2-TZVP basis set does not bring any significant modification. We will thus rely in the following on the TDA-PBE0/def2-TZVP//B3LYP-D3(BJ)/6-31G* level. 


\subsection{Thermally Activated Delayed Fluorescence}

The field of Computational Spectroscopy involves not only the study of the absorption spectra, but it also benefits from knowing the energy of the lowest singlet and triplet excited-states, and the number and nature of the states involved in the processes of light absorption and emission, photoconductivity and electroluminescence. We will investigate here the possibilities opened up for a novel process of emission of these compounds from their singlet excited-state, despite they had been shown before to be highly nonemissive [31], through a novel Thermally Activated Delayed Fluorescence (TADF) mechanism, pioneered by Adachi et al. [35], in which (dark) triplet excitons may be upconverted to singlet states thus increasing the fluorescence quantum yield. Note that we are primarily interested in exploring materials design in the context of small nanorings, based on their N-doping, thus anticipating a line of study which could be further developed with other acceptor moieties in the future.

We note that via the conventional Prompt Fluorescence (PF) mechanism only up to $25 \%$ of the electrogenerated excitons may be harvested, with several strategies employed so far to exploit the non-emissive triplet states such as triplet-triplet annihilation. TADF relies instead on up-conversion of the $75 \%$ of missing excitons by forcing a Reversed InterSystem Crossing (RISC) mechanism $[80,81]$. Thus, with a combination of prompt and Delayed Fluorescence (DF) it is possible to ideally achieve a $100 \%$ of quantum efficiency. We consider D-A doped CPPs as suitable TADF candidates by evaluating a key condition for maximising their performance [34]: the lowest singlet and triplet excited-states must be close enough in energy (ideally resonant or at least separated by less than $0.3 \mathrm{eV}$ ) to thus enable a sponta- 
neous or weakly endothermic RISC process. We also emphasize that some other difficulties may arise in practice: (i) this process is possibly gated by dynamical (i.e. conformationally mediated) effects [82,83]; (ii) experimental suppression of the non-radiative pathways for excited states must be also maximised; (iii) the role played by the whole manifold of lowest-lying $\mathrm{S}_{n}$ and $\mathrm{T}_{n}$ excited-states is still under experimental investigation [84]. Nevertheless, and to the best of our knowledge, this possibility is yet to be explored in cyclic constructs and might be the topic of future research; thus we explore preliminary here the potential use of these molecules without further studying the influence of vibronic, dynamic, environmental or other related effects.

\subsubsection{Singlet-triplet energy gap and nature of the excited-states}

We first discuss the energy difference between the lowest singlet and triplet excited-states, $\Delta E^{S T}$ as depicted in Figure 3, which is directly related to the RISC rate in the form $k_{R I S C} \propto V_{S O C}^{2} \exp \left(-\frac{\Delta E^{S T}}{k_{B} T}\right)$ [85]. We neglect here the possible variations, expectedly small, for the spin-orbit coupling $V_{S O C}$ among the set of closely related compounds, and present the results of our calculations in Table 3 for the set of N-doped CPPs studied. As TADF experiments are normally performed in thin films, meaning that some relaxation of the conformational degrees of freedom is allowed, we have also calculated the adiabatic $\Delta E_{a}^{S T}$ values for some selected compounds taken as representative examples, and found a difference of less than $0.1 \mathrm{eV}$ between both $\Delta E_{v}^{S T}$ and $\Delta E_{a}^{S T}$ results: 0.16 and $0.25 \mathrm{eV}$, and 0.17 and $0.23 \mathrm{eV}$, for compounds 4 and 7 respectively.

We have found a $\Delta E_{v}^{S T}$ value of around $0.5 \mathrm{eV}$ for compounds 1-3, in 
perfect agreement with the similar behaviour of these systems with respect to [8]CPP. This gap is instead approximately halved for the set of compounds 4-8. This reduction is related (vide infra) to the spatial separation, and thus poor overlap, of the HOMO and LUMO corresponding orbitals, as it was qualitatively evidenced in Figure 5. However, for compound 9, which registers a larger overlap between these orbitals (see also Figure 5) the value increases again showing the marked relationship between these two quantities in agreement with other recent studies $[86,87]$.

To determine more quantitatively the nature of the excited-states calculated so far, we resort to a set of descriptors mostly employed within the context of Charge-Transfer (CT) excitations, that is, the extent of the electronhole distance $(\Delta \mathbf{r}$, in $\AA$ ) as measured by the weighted distance between the corresponding centroids of the orbitals [88-90]:

$$
\Delta \mathbf{r}=\frac{\sum_{i a} \kappa_{i a}^{2}\left|\left\langle\phi_{a}|\hat{\mathbf{r}}| \phi_{a}\right\rangle-\left\langle\phi_{i}|\hat{\mathbf{r}}| \phi_{i}\right\rangle\right|}{\sum_{i a} \kappa_{i a}^{2}},
$$

with $\phi_{i(a)}$ referred again to the set of occupied (virtual) orbitals, and $\kappa_{i a}$ the corresponding coefficients, for the involved TDA-based excitation. We also rely on the overlap between the detachment and attachment densities, $\phi_{S}[91,92]$, based on the rearrangement of the electronic density removed upon electronic excitations (i.e. the detachment density matrix) and housed in the excited state of interest (i.e. the attachment density matrix), using the NANCY_EX package for both $S_{1}$ and $T_{1}$ states. Note that these two metrics consider by definition all the orbitals involved in the electronic excitation, gathering the results in Table 3 .

We remind that some of us [33,82] and others [86] have found before a direct correlation between low $\Delta E^{S T}$ values and high (low) values of $\Delta \mathbf{r}\left(\phi_{S}\right)$, 
an issue that we would like to further corroborate here. Actually, Figure 9 displays the existing interplay (or correlation) between some of the variables monitored $\left(\Delta_{v} E^{S T}, \phi_{S}\left(S_{1}\right)\right.$, and $\left.\Delta \mathbf{r}\left(S_{1}\right)\right)$ and indicates how this is indeed the case. Similar correlation is obtained when plotting $\phi_{S}\left(S_{1}\right)$ and $\Delta \mathbf{r}\left(S_{1}\right)$ as a function of $\Delta_{v} E^{S T}$. Since $\phi_{S}$ has normalized limiting values, 0 (1) when the electronic transition is of charge-transfer (locally excited) character by nature, we can corroborate that: (i) $\phi_{S}$ values close to 0.5 , like those found for the $S_{1}$ excited-state of molecules $\mathbf{4 - 8}$, indicate a mixed character although being predominantly ${ }^{1} \mathrm{CT}$; and (ii) the $T_{1}$ excited-state of all molecules displays values of $\phi_{S}>0.5$, and thus with a more pronounced Locally-Excited (LE) character $\left({ }^{3} \mathrm{LE}\right)$. The $\Delta \mathbf{r}$ metrics involves the manifold of one-electron transitions contributing to the excited-state, with some cutoff $(\Delta \mathbf{r}>1.5-2.0 \AA)$ proposed before to distinguish a charge-transfer excitation from a localized one. This quantity is thus expected to give a better indication of the spatial confinement of the orbitals. Table 3 also includes the numerical values of $\Delta \mathbf{r}$, indicating a much more marked CT character for compounds 4-8 than for 1-3. Note also how the values of $\Delta \mathbf{r}\left(T_{1}\right)$ are normally lower than $\Delta \mathbf{r}\left(S_{1}\right)$ owing to the more confined character of triplet excitons. Overall, according to these results, we can reduce the set of potential TADF candidates to molecules 4-8 ideally.

We remind that, in addition to the exploration of these materials as emitters, the development of host materials acting as matrix is also relevant. Generally speaking, and besides other factors as chemical and thermal robustness, the host molecules must possess a high triplet energy level to minimize back energy transfer from the emitter to the host. Actually, comparing the $E_{v}\left(T_{1}\right)$ values provided in Table 3 for compounds 4-8, ranging between 1.9-2.2 eV, 
with the experimental triplet energy levels [81] of some traditional hosts as CBP (2.6 eV), TPBI $(2.65 \mathrm{eV})$, mCBP $(2.8 \mathrm{eV})$, or DPEPO $(3.0 \mathrm{eV})$, we can see how this condition would also match. Another energy alignment should arise between emitters and hosts: the former should have a higher HOMO (lower Ionization Potential) and a lower LUMO (higher Electron Affinity) than the latter. A general compilation of experimental IP and EA values for host materials, as is given in Ref. [81], provides roughly speaking the following limits: $5.5<\mathrm{IP}<6.5$ and $2.0<\mathrm{EA}<3.5 \mathrm{eV}$, which would approximately translate into the conditions $3.8<-\epsilon_{\mathrm{HOMO}}<4.6$ and $4.1<-\epsilon_{\mathrm{LUMO}}<6.5$ $\mathrm{eV}$, thanks to the linear relationship existing between calculated (B3LYP) HOMO/LUMO energies and experimental IPs/EAs [93]. Recovering now the HOMO and LUMO values of emitters 4-8 displayed in Figure 5, we can see how this is always the case, with HOMO (LUMO) energies being higher (lower) than the thresholds derived above.

\section{Conclusions}

The impact of chemical functionalisations of the CPP backbone was established though a thorough theoretical study, carefully analyzing the effect of forcing an intramolecular D-A structure upon substitution of phenylene rings by an acceptor moiety. First of all, the results quantitatively reproduced the available (but scarce) experimental data, and thus enabled suitable bracketing of the accuracy of the computational tools employed. Substantial electronic changes were observed upon substitution with strong N-doped acceptor groups, such as pyridinium and methylpyridinium, affecting also the lowest singlet and triplet excited states and their nature. We also studied how the optoelectronic properties evolve with the size of the nanohoop, and 
how they hold for other acceptor moieties as far as the molecular design principle is not longer altered.

The state-of-the-art quantum-chemical methods and tools employed for modelling these nanorings allowed us to predict remarkably low singlet-triplet energy gap, and wholly characterize the nature of the excited-state involved. Thus, this study also opens up new ways to exploit systems with cyclic topology as TADF molecular emitters for OLEDs applications, considering that the singlet-triplet energy difference is at least halved with respect to the pristine (undoped) parent compound, approaching values lower than the $0.3 \mathrm{eV}$ energy threshold, as well as other key energy alignments are matched. We hope this finding will stimulate further theoretical and experimental work within this cutting-edge domain.

\section{Acknowledgements}

We acknowledge the "Ministerio de Economía y Competitividad" of Spain and the "European Regional Development Fund" through project CTQ201455073-P, the E2TP-CYTEMA-SANTANDER program, the French national grant ANR-10-LABX-0042-AMADEus managed by the National Research Agency under the initiative of excellence IdEx Bordeaux programme (reference ANR-10-IDEX-0003-02), the Programme d'Excellence de la Région Wallonne (OPTI2MAT project), and the European Unions Horizon 2020 research and innovation program under Grant Agreement N. 646176 (EXTMOS project). Computational resources in Mons have been provided by the Con-

sortium des Équipements de Calcul Intensif (CÉCI), funded by the Fonds de la Recherche Scientifique de Belgique (F.R.S.-FNRS) under Grant no. 


\section{References}

[1] S. Chen, L. Deng, J. Xie, L. Peng, L. Xie, Q. Fan, W. Huang, Adv. Mater. 2010, 22, 5227-5239.

[2] Q. Meng, H. Dong, W. Hu, D. Zhu, J. Mater. Chem. 2011, 21, 1170811721.

[3] C. Raston, V. Sgobba, D. M. Guldi, J. Mater. Chem. 2008, 18, 153-157.

[4] K. M. Pelzer, S. B. Darling, Mol. Syst. Des. Eng. 2016, 1, 10-24.

[5] P. L. Burn, S. C. Lo, I. D. W. Samuel, Adv. Mater. 2007, 19, 1675-1688.

[6] B. K. An, J. Gierschner, S. Y. Park, Acc. Chem. Res. 2012, 45, 544-554.

[7] Y. Yomogida, H. Sakai, K. Sawabe, S. Gocho, S. Z. Bisri, H. Nakanotani, C. Adachi, T. Hasobe, Y. Iwasa, T. Takenobu, Org. Electron. 2013, 14, $2737-2742$.

[8] J. Liu, H. Zhang, H. Dong, L. Meng, L. Jiang, L. Jiang, Y. Wang, J. Yu, Y. Sun, W. Hu et al. Nat. Commun. 2015, 6, 10032.

[9] C. Zhang, P. Chen, W. Hu, Small 2016, 12, 1252-1294.

[10] J. W. Park, D. C. Shin, S. H. Park, Semicond. Sci. Technol. 2011, 26, 34002 .

[11] S. Y. Lee, T. Yasuda, Y. S. Yang, Q. Zhang, C. Adachi, Angew. Chem. Int. Ed. 2014, 53, 6402-6406. 
[12] A. Slodek, M. Filapek, E. Schab-Balcerzak, M. Grucela, S. Kotowicz, H. Janeczek, K. Smolarek, S. Mackowski, J. G. Malecki, A. Jedrzejowska et al. Eur. J. Org. Chem. 2016, 2016, 4020-4031.

[13] K. Tahara, Y. Tobe, Chem. Rev. 2006, 106, 5274-5290.

[14] S. Yamago, Y. Watanabe, T. Iwamoto, Angew. Chem. Int. Ed. 2010, 49, 757-759.

[15] S. Yamago, E. Kayahara, T. Iwamoto, Chem. Rec. 2014, 14, 84-100.

[16] P. J. Evans, E. R. Darzi, R. Jasti, Nat. Chem. 2014, 6, 404-408.

[17] P. A. Denis, M. Yanney, New J. Chem. 2015, 40, 202-208.

[18] M. R. Golder, C. E. Colwell, B. M. Wong, L. N. Zakharov, J. Zhen, R. Jasti, J. Am. Chem. Soc. 2016, 138, 6577-6582.

[19] J. C. Sancho-García, M. Moral, A. J. Pérez-Jiménez, J. Phys. Chem. C 2016, 120, 9104-9111.

[20] F. E. Golling, M. Quernheim, M. Wagner, T. Nishiuchi, K. Müllen, Angew. Chem. Int. Ed. 2014, 53, 1525-1528.

[21] F. E. Golling, S. Osella, M. Quernheim, M. Wagner, D. Beljonne, K. Müllen, Chem. Sci. 2015, 6, 7072-7078.

[22] Y. Luan, H. Cong, Synlett 2017, 28, A-F.

[23] S. M. Bachrach, D. Stück, J. Org. Chem. 2010, 75, 6595-6604.

[24] T. Kuwabara, J. Orii, Y. Segawa, K. Itami, Angew. Chem. Int. Ed. 2015, 54, 9646-9649. 
[25] M. Ball, B. Fowler, P. Li, L. A. Joyce, F. Li, T. Liu, D. Paley, Y. Zhong, H. Li, S. Xiao, F. Ng, M. L. Steigerwald, C. Nuckolls, J. Am. Chem. Soc. 2015, 137, 9982-9987.

[26] H. Thakellapalli, B. Farajidizaji, T. W. Butcher, N. G. Akhmedov, B. V. Popp, J. L. Petersen, K. K. Wang, Org. Lett. 2015, 17, 3470-3473.

[27] H. Ito, Y. Mitamura, Y. Segawa, K. Itami, Angew. Chem. Int. Ed. 2015, 54, 159-163.

[28] A. Bandyopadhyay, S. K. Pati, Phys. Chem. Chem. Phys. 2016, 18, 20682-20690.

[29] B. Farajidizaji, H. Thakellapalli, S. Li, C. Huang, N. N. Baughman N. G. Akhmedov, B. V. Popp, J. L. Petersen, K. K. Wang, Chem. Eur. J. 2016, 22, 16420-16424.

[30] S. Nishigaki, M. Fukui, H. Sugiyama, H. Uekusa, S. Kawauchi, Y. Shibata, K. Tanaka, Chem. Eur. J. 2017, 23, 7227-7231.

[31] E. R. Darzi, E. S. Hirst, C. D. Weber, 1. N. Zakharov, M. C. Lonergan, R. Jasti, ACS Cent. Sci. 2015, 1, 335-342.

[32] B. Milián-Medina, J. Gierschner, Org. Electron. 2012, 13, 985-991.

[33] M. Moral, L. Muccioli, W.-J. Son, Y. Olivier, J. C. Sancho-García, J. Chem. Theory Comput. 2015, 11, 168-177.

[34] Y. Tao, K. Yuan, T. Chen, P. Xu, H. Li, R. Chen, C. Zheng, L. Zhang, W. Huang, Adv. Mater. 2014, 26, 7931-7958.

[35] Q. Zhang, B. Li, S. Huang, H. Nomura, H. Tanaka, C. Adachi, Nat. Photon. 2014, 8, 1-7. 
[36] M. Aydemir, G. Haykir, A. Battal, V. Jankus, S. K. Sugunan, F. B. Dias, H. Al-Attar, F. Türksoy, M. Tavasli, A. P. Monkman, Org. Electron. 2016, 30, 149-157.

[37] P. Data, P. Pander, M. Okazaki, Y. Takeda, S. Minakata, A. P. Monkman, Angew. Chem. Int. Ed. 2016, 55, 5739-5744.

[38] Gaussian 09, Revision D.03, M. J. Frisch, G. W. Trucks, H. B. Schlegel, G. E. Scuseria, M. A. Robb, J. R. Cheeseman, G. Scalmani, V. Barone, B. Mennucci, G. A. Petersson, H. Nakatsuji, M. Caricato, X. Li, H. P. Hratchian, A. F. Izmaylov, J. Bloino, G. Zheng, J. L. Sonnenberg, M. Hada, M. Ehara, K. Toyota, R. Fukuda, J. Hasegawa, M. Ishida, T. Nakajima, Y. Honda, O. Kitao, H. Nakai, T. Vreven, J. A. Montgomery, Jr., J. E. Peralta, F. Ogliaro, M. Bearpark, J. J. Heyd, E. Brothers, K. N. Kudin, V. N. Staroverov, R. Kobayashi, J. Normand, K. Raghavachari, A. Rendell, J. C. Burant, S. S. Iyengar, J. Tomasi, M. Cossi, N. Rega, J. M. Millam, M. Klene, J. E. Knox, J. B. Cross, V. Bakken, C. Adamo, J. Jaramillo, R. Gomperts, R. E. Stratmann, O. Yazyev, A. J. Austin, R. Cammi, C. Pomelli, J. W. Ochterski, R. L. Martin, K. Morokuma, V. G. Zakrzewski, G. A. Voth, P. Salvador, J. J. Dannenberg, S. Dapprich, A. D. Daniels, Ö. Farkas, J. B. Foresman, J. V. Ortiz, J. Cioslowski, and D. J. Fox, Gaussian, Inc., Wallingford CT, 2009.

[39] A. R. Allouche, J. Comput. Chem. 2011, 32, 174-182.

[40] T. Lu, F. Chen, J. Comput. Chem. 2011, 33, 580-592.

[41] T. Etienne, X. Assfeld, A. Monari, J. Chem. Theory Comput. 2014, 10, 3896-3905. 
[42] T. Etienne, X. Assfeld, A. Monari, J. Chem. Theory Comput. 2014, 10, 3906-3914.

[43] V. Barone, M. Cossi, J. Phys. Chem. A 2001, 102, 1995-2001.

[44] M. Cossi, N. Rega, G. Scalmani, V. Barone, J. Comput. Chem. 2003, 24, 669-681.

[45] D. D. Méndez-Hernández, J. G. Gillmore, L. A. Montano, T. A. Moore, A. L. Moore, V. Mujica, J. Phys. Org. Chem. 2015, 28, 320-328.

[46] J. Tomasi, B. Mennucci, R. Cammi, Chem. Rev. 2005, 105, 2999-3093.

[47] F. Weigend, R. Ahlrichs, Phys. Chem. Chem. Phys. 2005, 7, 3297-3305.

[48] F. Weigend, Phys. Chem. Chem. Phys. 2006, 8, 1057-1065.

[49] Y. Zhao, D. G. Truhlar, Theor. Chem. Acc. 2008, 120, 215-241.

[50] A. D. Becke, J. Chem. Phys. 1993, 98, 1372-1376

[51] V. Barone, C. Adamo, Chem. Phys. Lett. 1994, 224, 432-438.

[52] J. D. Chai, M. Head-Gordon, J. Chem. Phys. 2008, 128, 084106.

[53] S. Grimme, J. Antony, S. Ehrlich, H. Krieg, J. Chem. Phys. 2010, 132, 154104 .

[54] S. Grimme, S. Ehrlich, L. Goerigk, J. Comput. Chem. 2011, 32, 14561465.

[55] B. Moore, A. Charaf-Eddim, A. Planchat, C. Adamo, J. Autschbach, D. Jacquemin, J. Chem. Theory Comput. 2014, 10, 4599-4608.

[56] D. Jacquemin, C. Adamo, Top. Curr. Chem. 2015, 368, 347-376. 
[57] C. Adamo, V. Barone, J. Chem. Phys. 1999, 110, 6158-6170.

[58] S. Grimme, Chem. Phys. Lett. 1996, 259, 128-137.

[59] S. Hirata, M. Head-Gordon, Chem. Phys. Lett. 1999, 314, 291-299.

[60] M. J. G. Peach, M. J. Williamson, D. J. Tozer, J. Chem. Theory Comput. 2011, 7, 3578-3585.

[61] M. J. G. Peach, D. J. Tozer, J. Phys. Chem. A 2012, 116, 9783-9789.

[62] M. J. G. Peach, N. Warner, D. J. Tozer, Mol. Phys. 2013, 111, 12711274 .

[63] S. Grimme, J. Chem. Phys. 2013, 138, 244104.

[64] J. Xia and R. Jasti, Angew. Chem. Int. Ed. 2012, 51, 2474-2476.

[65] C. Ambrosch-Draxl, J. A. Majewski, P. Vogl, J. Leising, Phys. Rev. B 1995, 51, 9668-9676.

[66] Y. Olivier, L. Muccioli, C. Zannoni, ChemPhysChem 2014, 15, 13451355

[67] M. Kertesz, C. H. Choi, S. Yang, Chem. Rev. 2005, 105, 3448-3481.

[68] J. Gierschner, J. Cornil, H.-J. Egelhaaf, Adv. Mater. 2007, 19, 173-191.

[69] S. Tretiak, K. Igumenshchev, V. Chernyak, Phys. Rev. B 2005, 71, 033201.

[70] J. C. Sancho-García, A. J. Pérez-Jiménez, Phys. Chem. Chem. Phys. 2007, 9, 5874-5879.

[71] J. C. Sancho-García, C. Adamo, A. J. Pérez-Jiménez, Theor. Chem. Acc. 2016, 135, 25. 
[72] Y. Segawa, A. Fukuzawa, S. Matsuura, H. Omachi, S. Yamaguchi, S. Irle, K. Itami, Org. Biomol. Chem. 2012, 10, 5979-5984.

[73] J. M. Van Raden, E. R. Darzi, L. N. Zakharov, R. Jasti, Org. Biomol. Chem. 2016, 14, 5721-5727.

[74] E. R. Darzi, R. Jasti, Chem. Soc. Rev. 2015, 44, 6401-6410.

[75] Y. Noguchi, O. Sugino, J. Chem. Phys. 2017, 146, 144304.

[76] B. M. Wong, J. Phys. Chem. C 2009, 113, 21921-21927.

[77] B. M. Wong, J. W. Lee, J. Phys. Chem. Lett. 2011, 2, 2702-2706.

[78] D. A. Hines, E. R. Darzi, E. S. Hirst, R. Jasti, P. V. Kamat, J. Phys. Chem. A 2015, 119, 8083-8089.

[79] A. J. Heeger, Adv. Mater. 2014, 26, 10-28.

[80] P. L. dos Santos, J. S. Ward, M. R. Bryce, A. P. Monkman, J. Phys. Chem. Lett. 2016, 7, 3341-3346.

[81] M. Y. Wong, E. Zysman-Colman, Adv. Mater. 2017, 29, 1605444.

[82] Y. Olivier, M. Moral, L. Muccioli, J. C. Sancho-García, J. Mater. Chem. C 2017, 5, 5718-5729.

[83] M. K. Etherington, F. Franchello, J. Gibson, T. Northey, J. Santos, J. S. Ward, H. F. Higginbotham, P. Data, A. Kurowska, P. L. Dos Santos, D. R. Graves, A. S. Batsanov, F. B. Dias, M. R. Bryce, T. J. Penfold, and A. P. Monkman, Nat. Commun. 2017, 8, 14987.

[84] J. Gibson, A. P. Monkmann, and T. J. Penfold, ChemPhysChem 2016, 17, 2956-2961. 
[85] F. B. Dias, T. J. Penfold, A. P. Monkmann, Methods Appl. Fluoresc. 2017, 5, 012001 .

[86] T. J. Penfold, J. Phys. Chem. C 2015, 119, 13535-13544.

[87] M. Alipour, S. Damiri, ChemPhysChem 2017, 18, 480-487.

[88] C. A. Guido, P. Cortona, B. Mennucci, C. Adamo, J. Chem. Theory Comput. 2013, 9, 3118-3126.

[89] M. Ehara, R. Fukuda, C. Adamo, I. Ciofini. J. Comput. Chem. 2013, 34, 2498-2501.

[90] C. A. Guido, P. Cortona, C. Adamo, J. Chem. Phys. 2014, 140, 104101.

[91] M. Head-Gordon, A. M. Grana, D. Maurice, C. A. White, J. Phys. Chem. 1995, 99, 14261-14270.

[92] A. Dreuw, M. Head-Gordon, Chem. Rev. 2005, 105, 4009-4037.

[93] C.-G. Zhan, J. A. Nichols, D. A. Dixon, J. Phys. Chem. A 2003, 107, 4184-4195. 
- Table 1. Bond Length Alternation (BLA, in Å), calculated as BLA $=\frac{1}{2}\left[\left(r_{1}+r_{1}^{\prime}\right)-\left(r_{2}+r_{2}^{\prime}\right)\right]$, and dihedral angles $(\theta$, in degrees $)$ along the backbone of the selected N-doped CPPs, also compared with the pristine [8]CPP case. A positive (negative) sign for the angles implies a (anti-)clockwise orientation. All values obtained from the optimized geometries at the B3LYP-D3(BJ)/6-31G* level. The phenyl rings 1 and 3 carry the nitrogen functionality in all cases.

- Table 2. Calculated vertical (lowest) singlet excitation energies (in $\mathrm{eV}$ ) of compounds N-methylaza-[8] $\mathrm{CPP}^{+}$and N,N-dimethyl-1,15-diaza$[8] \mathrm{CPP}^{2+}$, with different functionals and basis sets.

- Table 3. Calculated vertical (lowest) singlet and triplet excitation energies (in eV), and their energy difference, for the set of N-doped CPPs 1 to 9. The oscillator strenghts are given in parentheses. The values of $\Delta \mathbf{r}$ (in $\AA$ ) and $\phi_{S}$ for each excited-state are also given. All values obtained at the TDA-PBE0/def2-TZVP level, unless for $\phi_{S}$ in which the basis set used was the $6-31 \mathrm{G}^{*}$ one. 


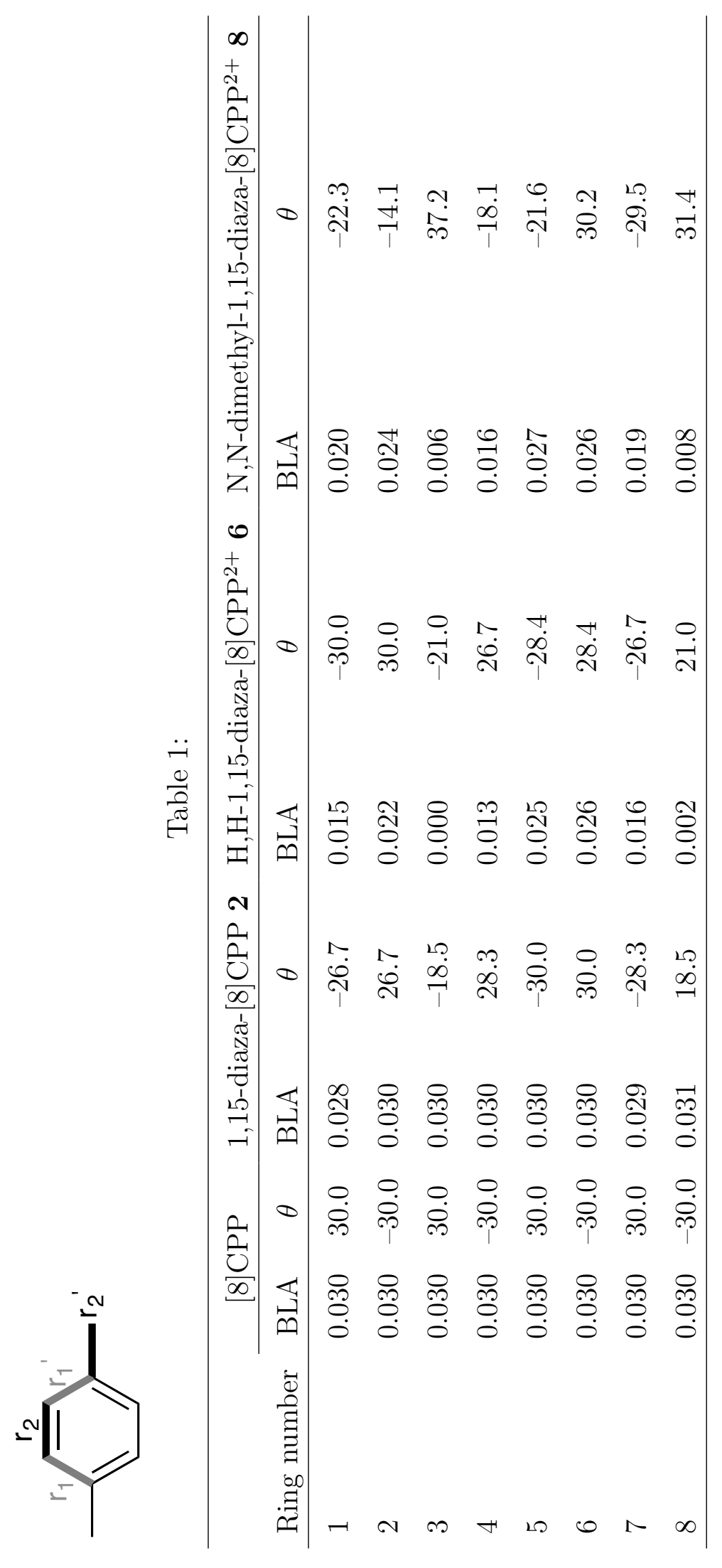


Table 2:

\begin{tabular}{|c|c|c|}
\hline Method $^{\mathrm{a}}$ & N-methylaza- $[8] \mathrm{CPP}^{+} \mathbf{7}$ & N,N-dimethyl-1,15-diaza- $[8] \mathrm{CPP}^{2+} \mathbf{8}$ \\
\hline TDA-M06-L/6-31G* & 1.54 & 1.52 \\
\hline TDA-B3LYP/6-31G* & 2.20 & 2.05 \\
\hline TDA-PBE0/6-31G* & 2.41 & 2.23 \\
\hline TDA-PBE0/def2-TZVP & 2.41 & 2.21 \\
\hline TDA- $\omega B 97 X D / 6-31 G^{*}$ & 3.90 & 3.54 \\
\hline TDA-M06-HF/6-31G* & 4.46 & 4.18 \\
\hline Experimental values ${ }^{\mathrm{b}}$ & 2.69 & 2.24 \\
\hline
\end{tabular}


Table 3:

\begin{tabular}{lccccccc}
\hline Compound $^{\mathrm{a}}$ & $E_{v}\left(S_{1}\right)$ & $\Delta \mathbf{r}\left(S_{1}\right)$ & $\phi_{S}\left(S_{1}\right)$ & $E_{v}\left(T_{1}\right)$ & $\Delta \mathbf{r}\left(T_{1}\right)$ & $\phi_{S}\left(T_{1}\right)$ & $\Delta E_{v}^{S T}$ \\
\hline$[8] \mathrm{CPP}$ & $2.90(0.000)$ & 0.00 & - & 2.40 & 0.00 & - & 0.50 \\
$\mathbf{1}$ & $2.85(0.006)$ & 1.03 & 0.80 & 2.36 & 0.28 & 0.86 & 0.49 \\
$\mathbf{2}$ & $2.76(0.006)$ & 1.04 & 0.79 & 2.29 & 0.26 & 0.85 & 0.47 \\
$\mathbf{3}$ & $2.68(0.005)$ & 0.23 & 0.80 & 2.22 & 0.07 & 0.85 & 0.46 \\
$\mathbf{4}$ & $2.25(0.088)$ & 5.92 & 0.46 & 2.00 & 3.22 & 0.68 & 0.25 \\
$\mathbf{5}$ & $2.23(0.065)$ & 5.60 & 0.49 & 1.98 & 3.10 & 0.69 & 0.25 \\
$\mathbf{6}$ & $2.21(0.018)$ & 4.32 & 0.56 & 1.94 & 2.75 & 0.70 & 0.27 \\
$\mathbf{7}$ & $2.41(0.040)$ & 5.96 & 0.46 & 2.18 & 3.37 & 0.70 & 0.23 \\
$\mathbf{8}$ & $2.21(0.006)$ & 4.77 & 0.53 & 1.98 & 3.06 & 0.69 & 0.23 \\
$\mathbf{9}$ & $2.64(0.031)$ & 1.57 & 0.70 & 2.24 & 0.65 & 0.80 & 0.40 \\
\hline
\end{tabular}

${ }^{a}$ At the B3LYP-D3(BJ)/6-31G* optimized geometries and with DCM as solvent. 
- Figure 1. General strategy affording donor-acceptor nanohoops of cyclic topology.

- Figure 2. Nanohoop classification (top) and numbering (bottom) of N-substituted nanohoops: aza-[8]CPP 1; 1,15-diaza-[8]CPP 2; 1,15,31triaza-[8]CPP 3; H-aza-[8] $\mathrm{CPP}^{+} \mathbf{4}$; H-1,15-diaza-[8] $\mathrm{CPP}^{+}$5; H,H-1,15diaza-[8] $\mathrm{CPP}^{2+} \mathbf{6}$; N-methylaza- $[8] \mathrm{CPP}^{+} \mathbf{7}$; N,N-dimethyl-1,15-diaza[8] $\mathrm{CPP}^{2+} \mathbf{8} ; \mathrm{N}, \mathrm{N}, \mathrm{N}$-trimethyl-1,15,31-triaza-[8] $\mathrm{CPP}^{3+} \mathbf{9}$.

- Figure 3. Sketch of the main excited-states processes studied along this work. Note that the curves for the $S_{1}$ and $T_{1}$ excited-states are exaggeratedly shifted for the sake of clarity.

- Figure 4. Bond Length Alternation (BLA, in $\AA$ ), calculated as BLA = $\frac{1}{2}\left[\left(r_{1}+r_{1}^{\prime}\right)-\left(r_{2}+r_{2}^{\prime}\right)\right]$, along the phenyl rings of (top) 1,15-diaza[8]CPP 2, (middle) H,H-1,15-diaza-[8] $\mathrm{CPP}^{2+} \mathbf{6}$ and (bottom) N,N-dimethyl1,15-diaza-[8] $\mathrm{CPP}^{2+} \mathbf{8}$, calculated at various levels of theory and with the $6-31 \mathrm{G}^{*}$ basis set. The phenyl rings 1 and 3 carry the nitrogen functionality in all cases.

- Figure 5. From left to right: Isocontour plots and energy values (in acetonitrile) of the HOMO and LUMO orbitals for [8] CPP and the set of N-doped CPPs $\mathbf{1}$ to $\mathbf{9}$. Red and blue for positive and negative signs of the lobes. All values obtained from the optimized geometries at the B3LYP-D3(BJ)/6-31G* level. 
- Figure 6. From left to right: Increasingly longer cyclic (top) and linear (bottom) N-substituted nanohoops aza- $[n] \mathrm{CPP}, \mathrm{H}$-aza- $[n] \mathrm{CPP}^{+}$, and N-methylaza- $[n] \mathrm{CPP}^{+}$

- Figure 7. Simulated UV-vis absorption spectra (in dichloromethane and at the TDA-B3LYP/6-31G* level) of N-doped nanohoops: (top) aza-[8]CPP 1; 1,15-diaza-[8]CPP 2; 1,15,31-triaza-[8]CPP 3; (middle) $\mathrm{H}$-aza-[8] $\mathrm{CPP}^{+}$4; H-1,15-diaza- $[8] \mathrm{CPP}^{+}$5; H,H-1,15-diaza-[8] $\mathrm{CPP}^{2+}$ 6; (bottom) N-methylaza-[8] $\mathrm{CPP}^{+}$7; N,N-dimethyl-1,15-diaza-[8] $\mathrm{CPP}^{2+}$ 8; N,N,N-trimethyl-1,15,31-triaza-[8] $\mathrm{CPP}^{3+} \mathbf{9}$, also compared with the pristine $[8] \mathrm{CPP}$ case.

- Figure 8. Major orbital transitions, arising from the HOMO-2 to the $\mathrm{L}(+2)$ window of orbitals, for the: (a) N-methylaza- $[8] \mathrm{CPP}^{+} \mathbf{7}$, and (b) $\mathrm{N}, \mathrm{N}$-dimethyl-1,15-diaza-[8] $\mathrm{CPP}^{2+} \mathbf{8}$ systems, calculated at the TDAB3LYP/6-31G* level.

- Figure 9. Interplay between the calculated $\Delta r$ (in $\AA$ ) and $\phi_{S}$ values, and the $\Delta E_{v}^{S T}$ (in eV) energy difference, at the TDA-PBE0/def2-TZVP level. The solid lines are a guide to the eye. 


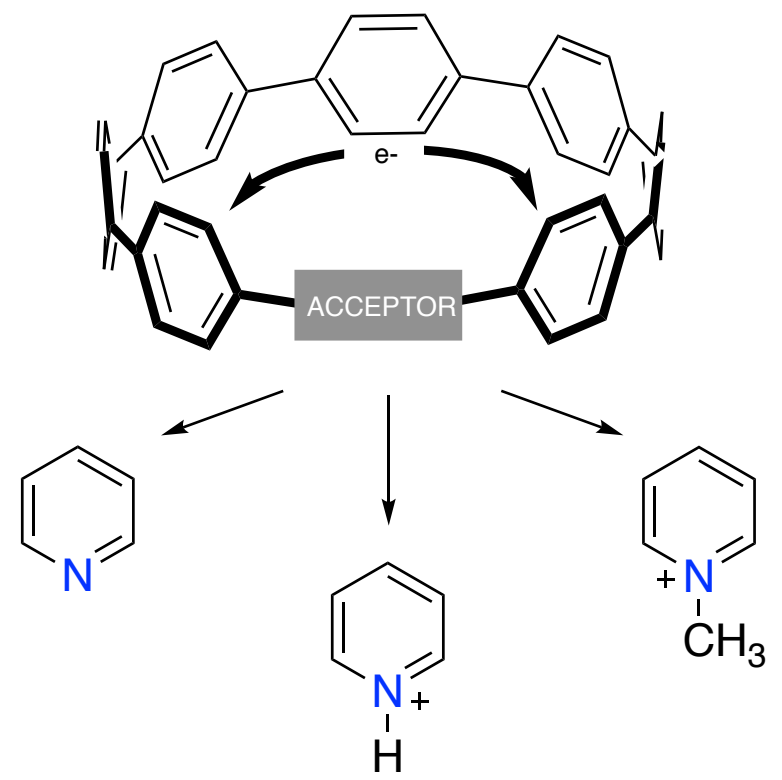

Figure 1. 

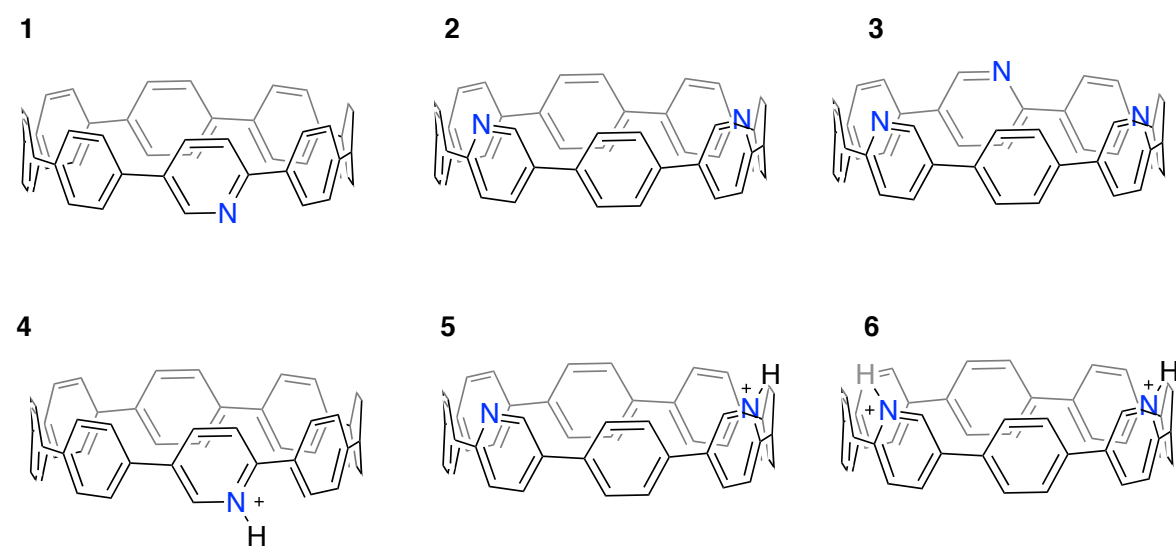

6
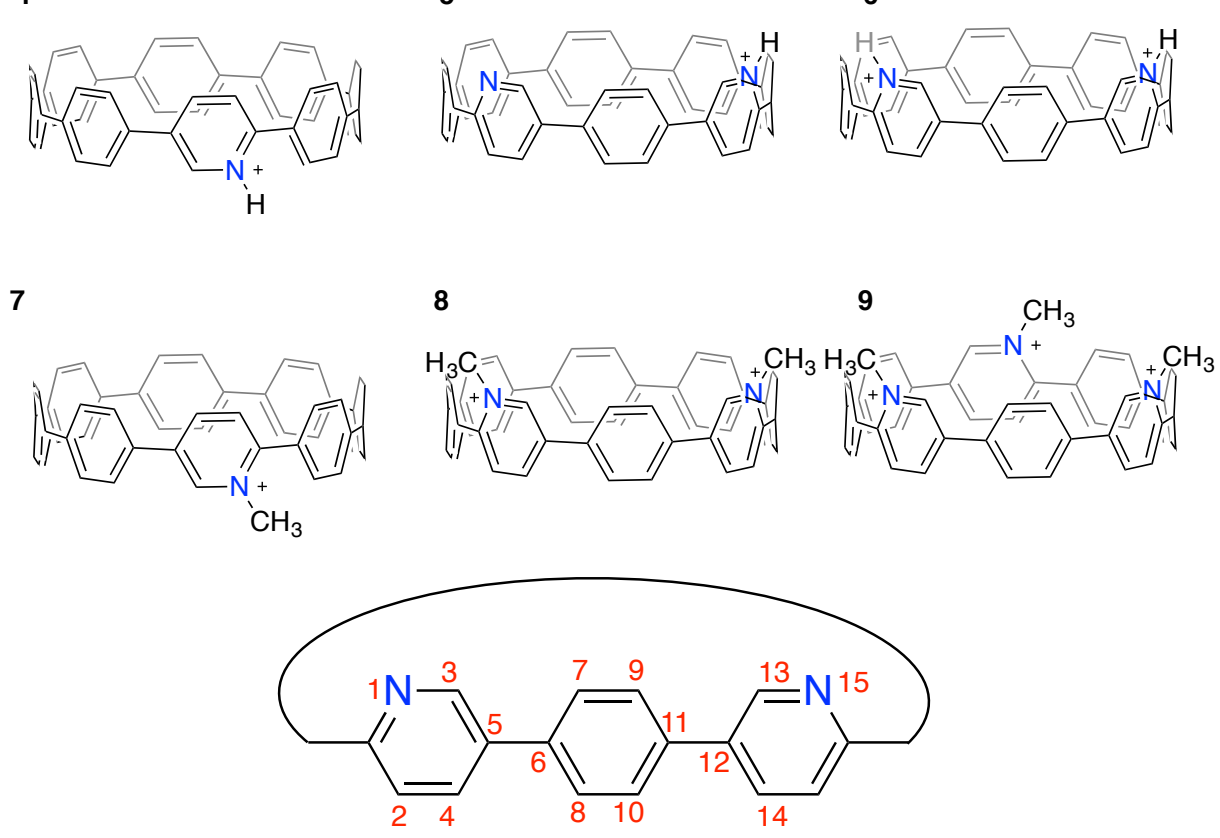

Figure 2. 


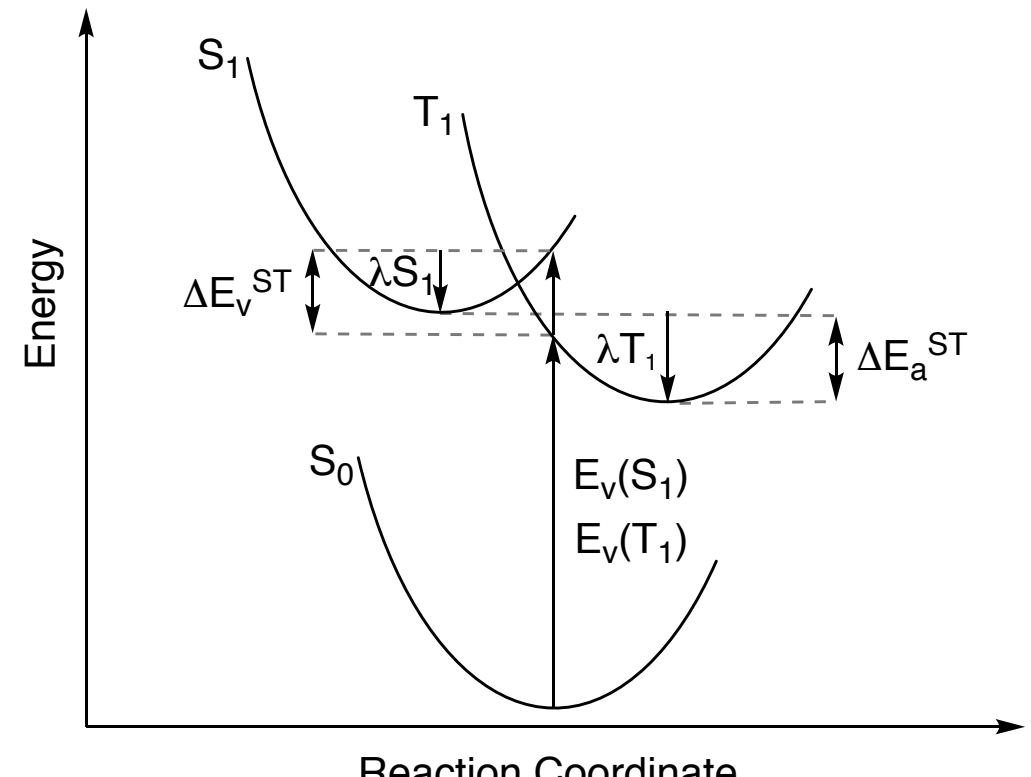

Figure 3. 

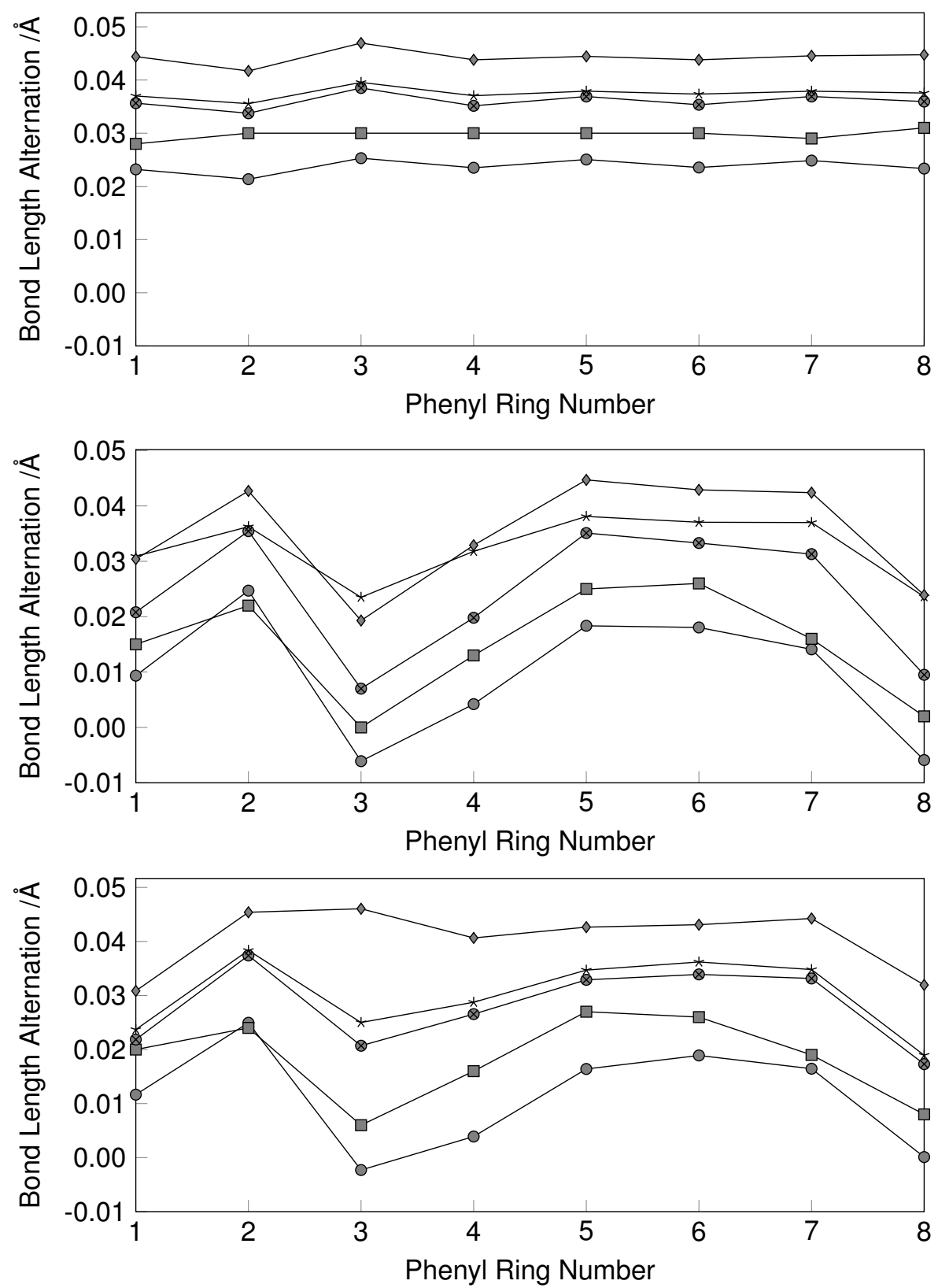

$\multimap-$ MO6-L $\square-$ B3LYP-D3(BJ) $ه-$ M06-2X * wB97XD $\multimap$ M06-HF

Figure 4. 


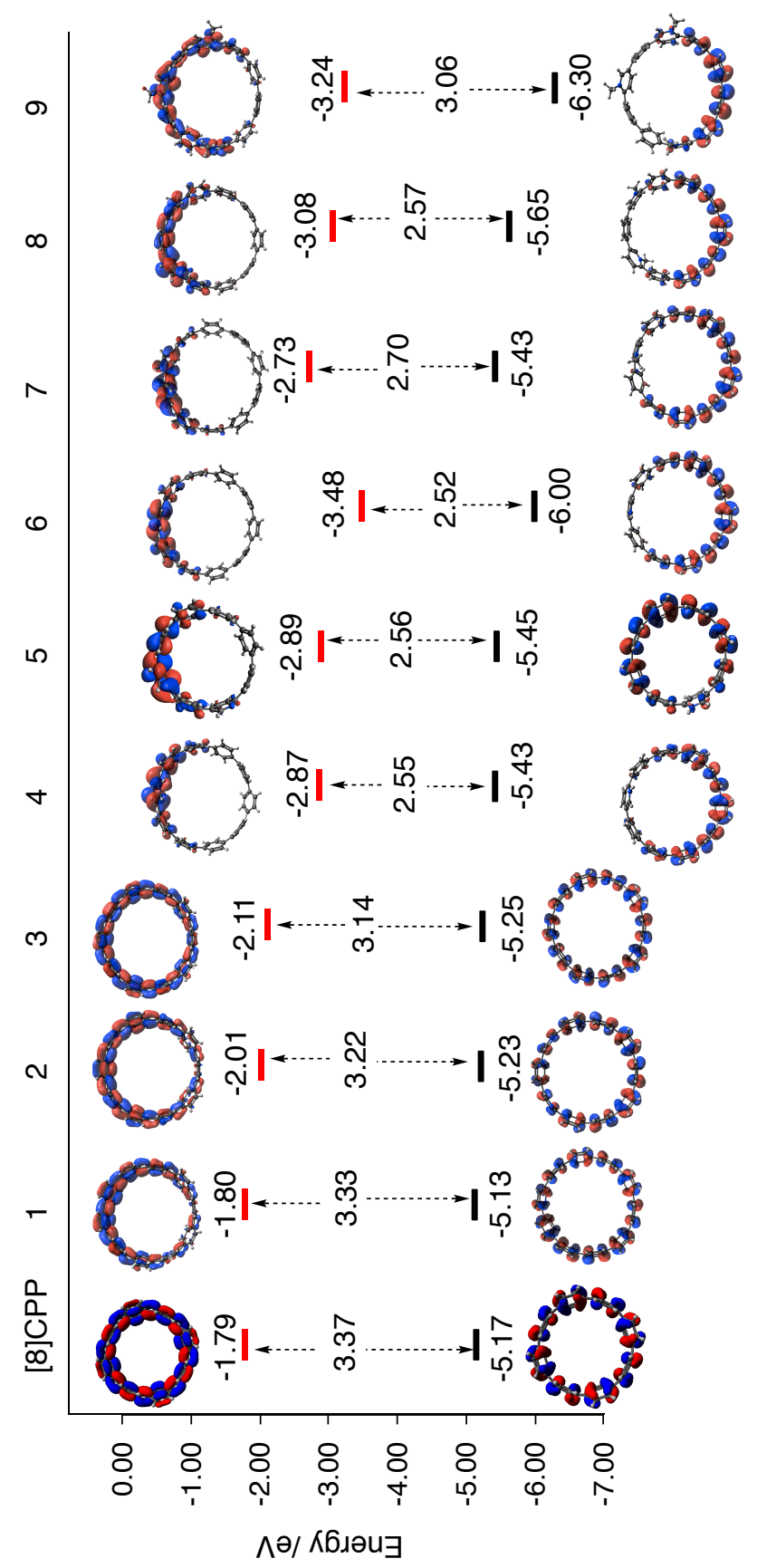

Figure 5. 

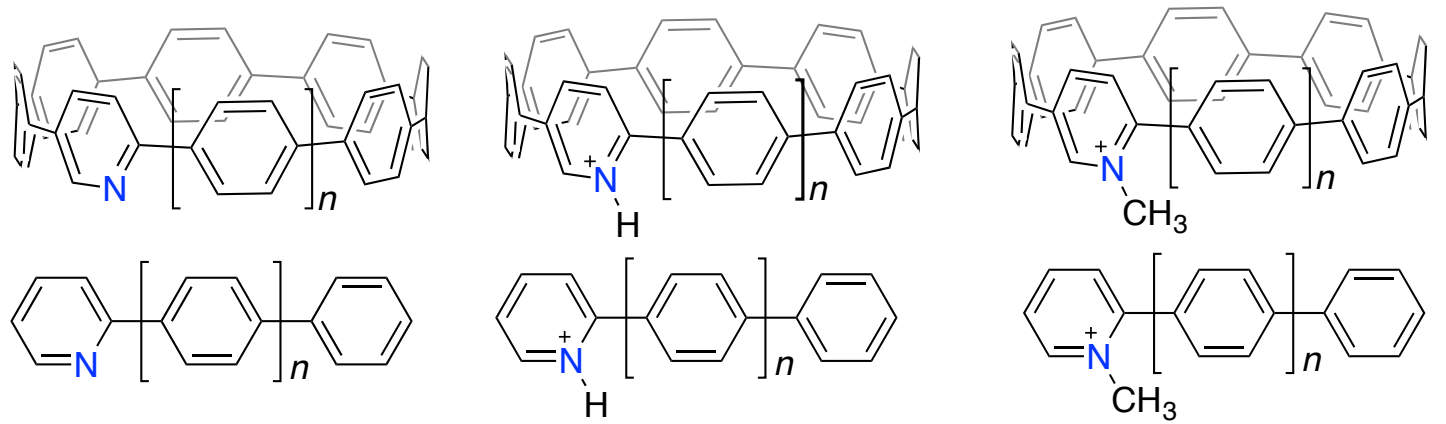

Figure 6. 

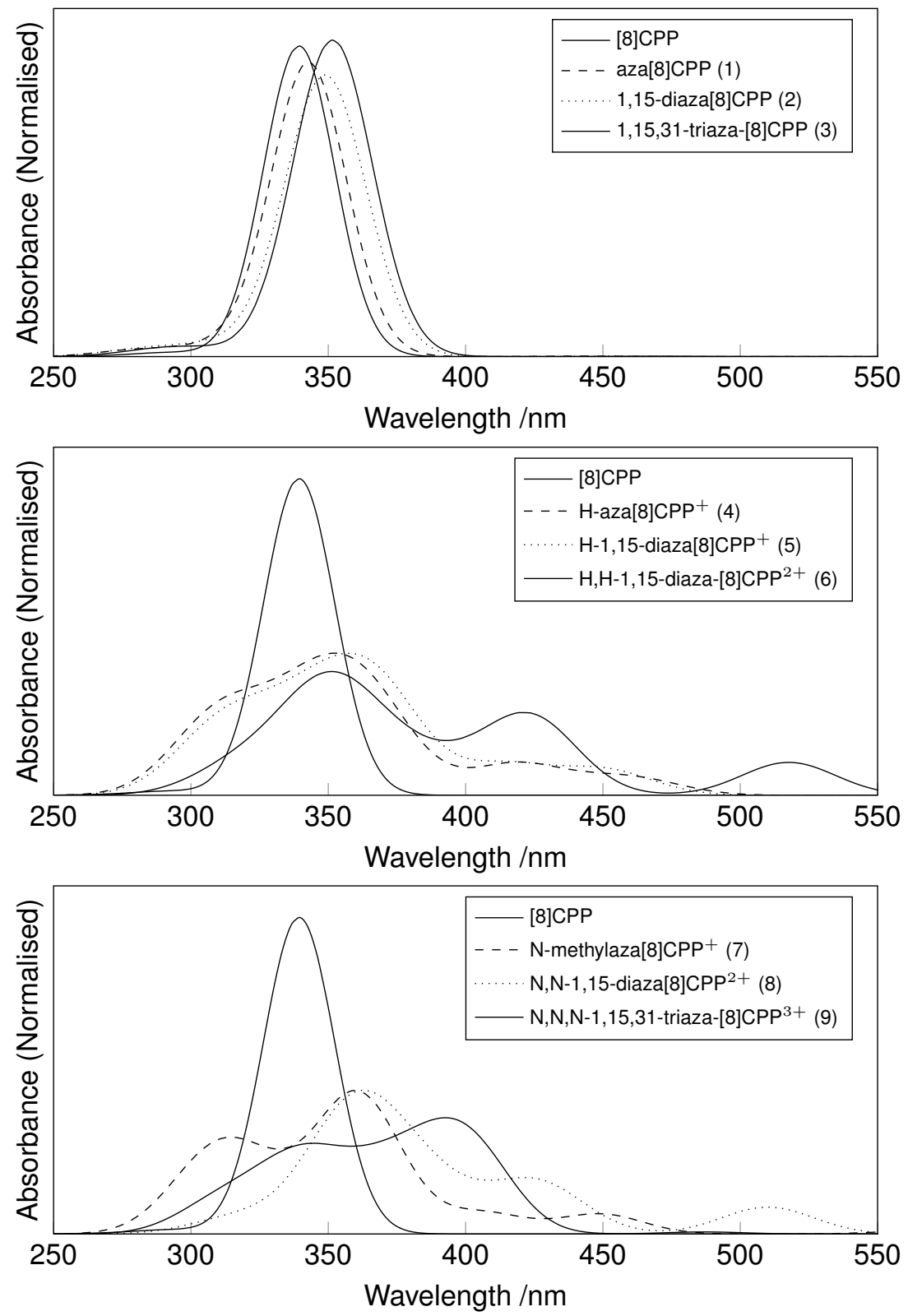

Figure 7 . 
a)

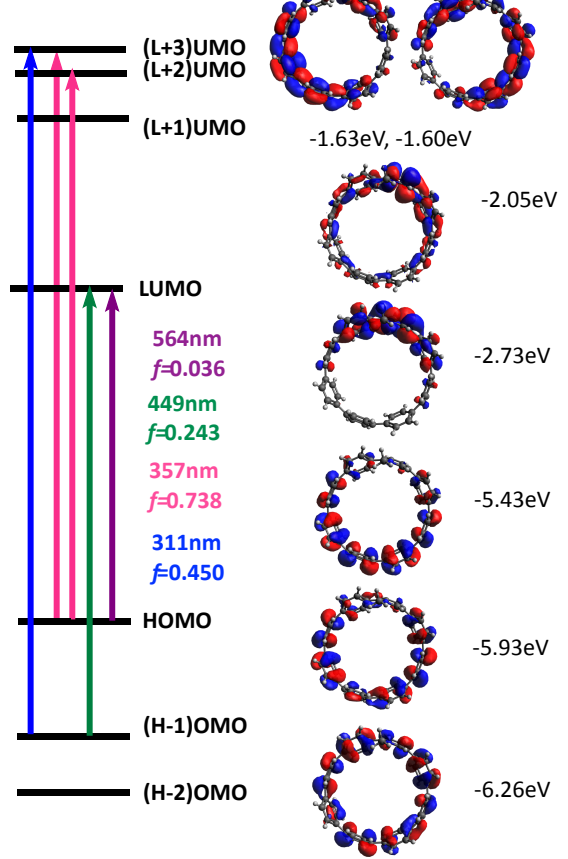

b)

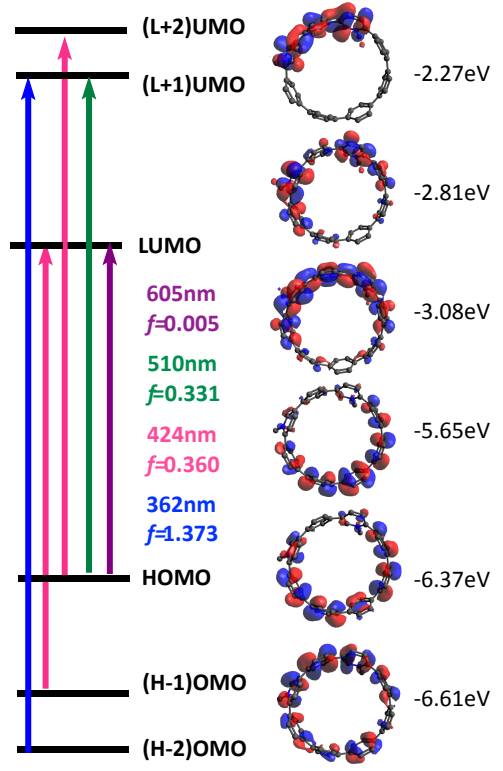

Figure 8. 


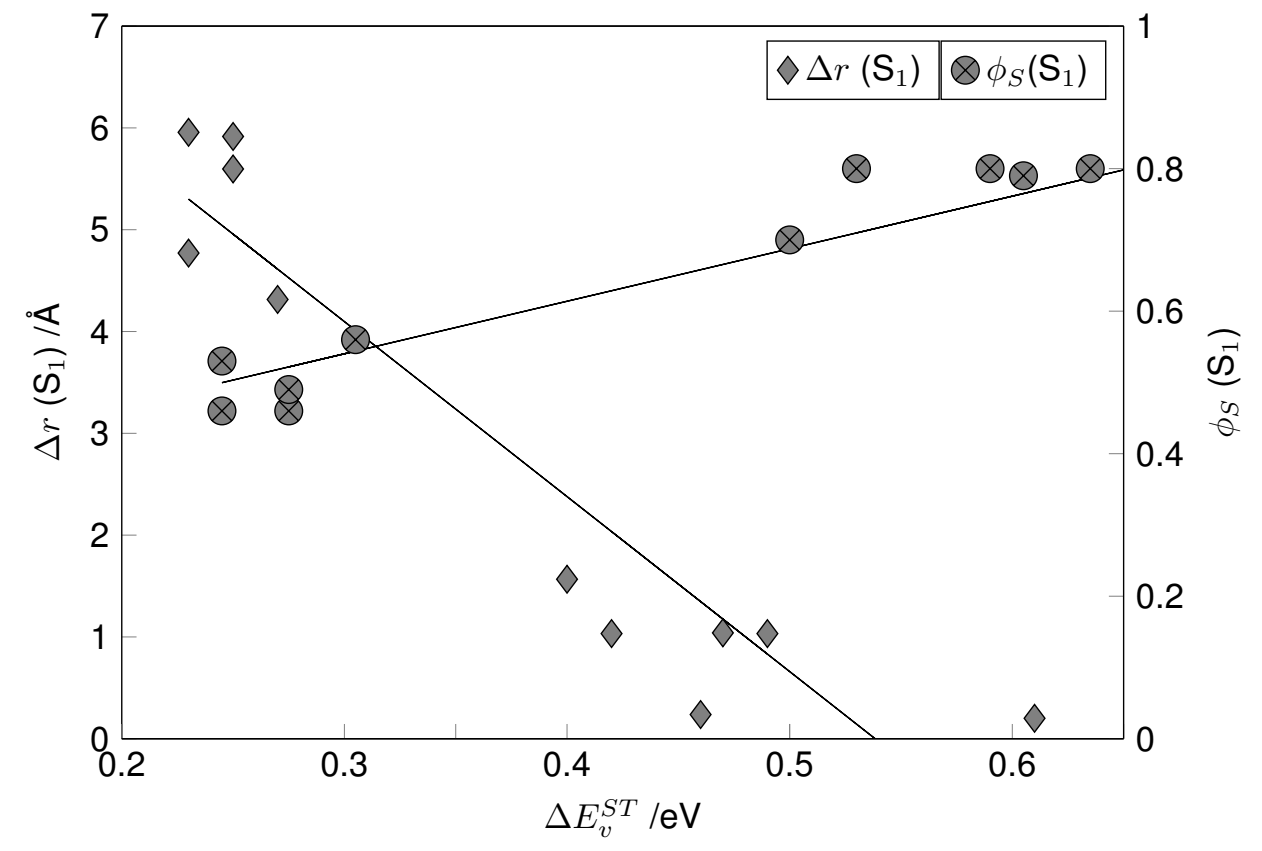

Figure 9. 